\title{
Analysis of the Critical Line Network for the van der Waals Equation at the van Laar Point
}

\author{
Paul H. E. Meijer, ${ }^{1}$ A. H. M. Levelt, ${ }^{2}$ and B. R. Miller $^{3}$
}

Received May 27, 1992; final October 26, 1992

The density-density plot of the critical lines of the van der Waals equation at the van Laar point is analyzed through its algebraic properties. It is shown that this curve is an irreducible expression of the fifth degree of genus one. In addition, we show the topology of the second branch, i.e., the $T=0$ solution, which will interact with the first branch as soon as the energy parameters are slightly different from the van Laar values. Finally, we analyze the behavior of the van der Waals equation near the point at which liquid-liquid separation takes place.

KEY WORDS: Critical lines; van der Waals equation; binary mixture; binodals; spinodals; double points; van Laar point; tricritical point; Gibbs function; liquid-liquid mixing.

\section{INTRODUCTION}

A given equation of state for binary fluid mixtures (liquid-liquid, gas-gas, and all forms in between) leads to a set of critical lines. Two well-known examples are the studies of Scott and van Konynenburg ${ }^{(1)}$ for the van der Waals equation and of Furman and Griffiths ${ }^{(2)}$ for the lattice gas and the van der Waals equation. In both cases the critical lines can be expressed in the form of polynomials in the density and concentration. Early work along these lines was done by Korteweg. ${ }^{(3)}$ The goal of this paper is to study the algebraic properties of these polynomials. Experience shows that certain special points of the critical line persist in more realistic equations of state, i.e., those containing more adjustable parameters in order to better

\footnotetext{
${ }^{1}$ Physics Department, Catholic University of America, Washington, District of Columbia 20064.

${ }^{2}$ Mathematical Institute, Katholieke Universiteit, 6525 ED Nijmegen, The Netherlands.

${ }^{3}$ Applied and Computational Mathematics Division, National Institute of Standards and Technology, Gaithersburg, Maryland 20899.
} 
describe experimental results. However, more general equations, such as the SPHCT (simplified perturbed hard-chain theory) equation used, for instance, by van Pelt and de Loos, ${ }^{(4)}$ may show additional special points, such as isolated critical points, ${ }^{(5)}$ for example.

The equations of state mentioned use a bilinear expression for the interaction energy, and hence contain three energy parameters, and the form of the critical lines depends on the choice of these parameters. The characteristics are classified by using the connectivities and other properties of the critical lines. Each type usually belongs to a certain range of energy parameters and this is most conveniently described by a map, referred to as the global map, which can be found in the literature. Parts of these critical line are stable, other parts unstable or metastable. For the algebraic study we ignore this distinction for the moment; not only that, to obtain a proper appreciation of the structure, it is advantageous to consider the curves outside the range of physical values; the "paraphysical" region, so to speak.

\section{ALGEBRAIC ANALYSIS}

In 1905 van Laar ${ }^{(6)}$ (see also ref. 7) pointed out that the critical lines of the van der Waals equation for binary mixtures do have a crossing point (i.e., a point at which the critical line intersects itself) in the volume versus concentration projection, provided the energy parameters are properly chosen. In this calculation he presumed that the geometrical mean condition for the intermolecular interaction was obeyed. It can be shown that the crossing point in this case is also a tricritical point. If the point at which the critical line intersects itself is also a tricritical point, it is called a van Laar point. Although the van Laar point fulfills also the geometrical mean condition in the van der Waals case, this is not the case for other equations of state. ${ }^{(8)}$ Van Laar was able to derive the condition for this crossing point algebraically by systematically reducing the relation between the various polynomials to a second-order equation, leading to a set of energy parameters given below. The importance of this special crossing point has been explained by Meijer. ${ }^{(9,10)}$ The point can be used as a reference for series expansions around this fulcrum.

In order to realize the van Laar condition, the energy parameters have to be properly chosen and for the van der Waals equation this fixes their values.

One should distinguish between the plot of a critical line and the so-called master or global diagram. The term master diagram was coined by van Konynenburg and Scott, ${ }^{(1)}$ while Furman and Griffiths ${ }^{(2)}$ use the expression global diagram. This type of diagram is convenient for three- 
parameter systems and the values of the parameters are used for the axes. Conditions on the parameters create a line or a point, depending on the number of conditions. For a system to have a tricritical point, one condition is required, hence the locus in the master diagram is a line. For a system to have a van Laar point in the critical line diagram, two conditions are required, hence the locus is represented by a point in the master diagram. Unfortunately, this point in the master diagram is also sometimes called the van Laar point.

The critical line structure corresponding to the van Laar point in the master diagram can be expressed in the form of a polynomial with respect to a limited field, in the mathematical sense of the word (i.e., a polynomial in the density and concentration variables has coefficients of the form $r_{0}+$ $r_{1} \sqrt{2}+r_{2} \sqrt{3}+r_{3} \sqrt{6} ; r_{0}, r_{1}, r_{2}$, and $r_{3}$ being rational numbers). We shall show that this polynomial does not admit further factorization. In the physical triangle (the borders of the triangle are determined by the restrictions on the concentration and the volume variable) the critical line consists of two, seemingly independent, intersecting branches (see Fig. 1: one branch goes from $C_{1}$ to $C_{0}$ and the other from $C_{2}$ to $C_{u}$ ); however, in this paper we prove that these two branches of the critical curve cannot be factorized. In addition, the possibility for parametrization of the critical curve is briefly mentioned. We have used the words "line" and "curve" interchangeably; physicists always use critical line, while mathematicians reserve the word line for a straight line only.

\section{CALCULATION}

The complete critical line of the van der Waals equation is obtained by elimination of $T$ from the following equations: the Hessian of the Helmholtz function for the generalized van der Waals equation, given by

$$
H=\alpha_{1} \alpha_{2}+\alpha_{1} \alpha_{0}+\alpha_{2} \alpha_{0}
$$

with

$$
\alpha_{1}=U_{1}-T / x_{1} ; \quad \alpha_{2}=U_{2}-T / x_{2} ; \quad \alpha_{0}=U_{0}-T\left(x_{0}+\sigma\right) / x_{0}^{2}
$$

and the condition for the locus of critical points given by

$$
\chi=\left(\frac{\partial H}{\partial x_{1}}-\frac{\partial H}{\partial x_{0}}\right) \alpha_{2}+\left(\frac{\partial H}{\partial x_{2}}-\frac{\partial H}{\partial x_{0}}\right) \alpha_{1}=0
$$

For $\sigma=1$ one obtains the van der Waals equation and for $\sigma=0$ the lattice gas equation is represented. In this paper we deal with the first case, unless 
mentioned otherwise. The first equation is quadratic in $T$ and can be written as a polynomial in $x_{1}, x_{2}$, and $x_{0}$, the second is of similar nature. The elimination of $T$ is described in ref. 1. Since the Eq. (1) is of the second degree in $T$, the elimination procedure makes use of both roots, one of which may be unphysical since the temperature needs to be real and positive. The constants $U_{1}, U_{2}$, and $U_{0}$ refer to the interaction between the particles. The variables $x_{1}$ and $x_{2}$ are the (relative) number densities of component 1 and component 2 of the binary mixture expressed in units of $b$, the covolume. The variable $x_{0}=1-x_{1}-x_{2}$ plays the role of the density of the holes in the lattice gas model; in the van der Waals case it is just an auxiliary variable. To obtain these equations, the interaction energy $E$ is expressed in the form

$$
E=-\left(a_{11} x_{1}^{2}+2 a_{12} x_{1} x_{2}+a_{22} x_{2}^{2}\right)
$$

by using

$$
\begin{aligned}
& U_{1}=2\left(a_{11}-a_{12}\right)=2(\Lambda-\zeta) /(1-\zeta) \\
& U_{2}=2\left(a_{22}-a_{12}\right)=2(\Lambda+\zeta) /(1-\zeta) \\
& U_{0}=2 a_{12}=2(1-\Lambda) /(1-\zeta)
\end{aligned}
$$

For convenience we also provide the expressions in terms of the interaction parameters $A$ and $\zeta$ used in ref. 1 , in reduced form: i.e., with $a_{11}=1$.

The accomplishment of van Laar $^{(6)}$ was to show that the critical curve has a mathematical double point, i.e., a point where the curve crosses itself, provided the following values are chosen for the interaction parameters:

$$
U_{0}=2 \phi_{1} \phi_{2} ; \quad U_{1}=2 \phi_{1}^{2}-U_{0} ; \quad U_{2}=2 \phi_{2}^{2}-U_{0}
$$

with $\phi_{1}=\frac{1}{2}(\sqrt{6}+\sqrt{2}-1) ; \phi_{2}=\frac{1}{2}(\sqrt{6}+\sqrt{2}+1)$.

Since the curve has a double point in the physical region $\left(0<x_{1}<1\right.$ and $0<x_{2}<1$, with $x_{1}+x_{2}<1$ ), it is convenient to place the origin of the coordinates at this crossing point. In an earlier paper one of us (Ref. 10) made a limited expansion around this point. We will now give the complete expression. Since the van Laar parameters introduce a field, in the mathematical sense, consisting of fractions as well as of fractions multiplied by $\sqrt{2}$, by $\sqrt{3}$, and by $\sqrt{6}$, one may greatly simplify the shift calculation if one introduces at the same time a rotation of coordinates. The complete transformation is

$$
\begin{aligned}
& u=x_{1}+x_{2}-\sqrt{3} / 3 \\
& v=\left(x_{1}-x_{2}\right) / \sqrt{2}-(3-\sqrt{3}) / 6
\end{aligned}
$$


The resulting polynomial describing the critical line of the van der Waals gas using the van Laar interaction parameters given by Eq. (3) now contains only $\sqrt{3}$. The curve is of the sixth degree. The leading terms were already given in ref. 10 . The complete expression for the critical line is

$$
\chi=P_{2}+P_{3}+P_{4}+P_{5}+P_{6}
$$

consisting of the following homogeneous parts:

$$
\begin{aligned}
P_{2}= & -\left(\frac{15}{4}+\frac{7}{4} \sqrt{3}\right) u^{2}-(6+2 \sqrt{3}) u+(3+3 \sqrt{3}) v^{2} \\
P_{3}= & -\left(6+\frac{7}{3} \sqrt{3}\right) u^{3}+\frac{1}{2}(21+25 \sqrt{3}) u^{2} v \\
& +(-15+3 \sqrt{3}) u v^{2}+\left(-6+\frac{10}{3} \sqrt{3}\right) v^{3} \\
P_{4}= & \left(32+\frac{55}{3} \sqrt{3}\right) u^{4}-\left(11+\frac{13}{3} \sqrt{3}\right) u^{3} v-\left(\frac{63}{2}+7 \sqrt{3}\right) u^{2} v^{2} \\
& -\left(2-\frac{50}{3} \sqrt{3}\right) u v^{3}+\left(-10+\frac{10}{3} \sqrt{3}\right) v^{4} \\
P_{5}= & (16+8 \sqrt{3}) u^{5}-(76+52 \sqrt{3}) u^{4} v+(96+36 \sqrt{3}) u^{3} v^{2} \\
& -(10+26 \sqrt{3}) u^{2} v^{3}+(10-2 \sqrt{3}) u v^{4} \\
P_{6}= & -(84+48 \sqrt{3}) u^{6}+(120+72 \sqrt{3}) u^{5} v-(72+36 \sqrt{3}) u^{4} v^{2} \\
& +12(1+\sqrt{3}) u^{3} v^{3}-3 u^{2} v^{4}
\end{aligned}
$$

Attempts to factorize these polynomials further, using computer algebra, failed at first. Computer algebra systems are good in factorizing polynomials with rational number coefficients. The computer algebra program Macsyma $^{4}$ has the option that one may declare a somewhat larger field. In this case the field chosen was one that included $\sqrt{3}$. With this option we found a separation of the above polynomial in the following two factors:

$$
F_{1}=2 v-2(\sqrt{3}+1) u-(1+\sqrt{3})
$$

and

$$
\begin{aligned}
F_{2}= & -3(3+4 \sqrt{3}) u^{2}-24 \sqrt{3} u v+36 v^{2} \\
& +(12+2 \sqrt{3}) u^{3}+(135+39 \sqrt{3}) u^{2} v-84 \sqrt{3} u v^{2}+20(3-\sqrt{3}) v^{3} \\
& +6(19+13 \sqrt{3}) u^{4}-4(72+27 \sqrt{3}) u^{3} v \\
& +9(5+13 \sqrt{3}) u^{2} v^{2}+12(-5+\sqrt{3}) u v^{3} \\
& +18[v-(1+\sqrt{3}) u]^{3} u^{2}
\end{aligned}
$$

${ }^{4}$ Identification of a commercial product does not imply recommendation or endorsement by NIST. 
The first factor represents a straight line. After transformation to the density variables, this becomes

$$
F_{1}=(\sqrt{2}-2-2 \sqrt{3}) x_{1}-(\sqrt{2}+2+\sqrt{3}) x_{2}
$$

Hence the line goes through the origin and from the slope one concludes that it never enters the density triangle, and hence plays no role. The second factor contains the essential structure of the critical line. Inside the triangle the polynomial represents two intersecting curves. Hence the question did arise whether further factorization might be possible. A necessary condition to further factorization is that the curves are not connected to each other somewhere outside the triangle. The simplest way to inspect this is to "zoom" outside the physical triangle. The asymptotic behavior is determined by the fifth-order terms which contain the factors $u=0$ (two times) and $v=(1+\sqrt{3}) u$ (three times); hence there may be five asymptotic directions and two, respectively three, are mutually parallel. The absence of complex roots guarantees that there is no connection between the two branches that were observed in the triangle.

Further analysis shows that the asymptotes $u=0$ belong to an isolated double point at infinity; consequently there are three parallel asymptotes. Their position is given by $(1+\sqrt{3}) u-v+c_{i}=0$ with $c_{1}=-1.272933030$, $c_{2}=1.366025404$, and $c_{3}=4.004983839$. If we include the point at infinity, which can be formally done by introducing homogeneous coordinates, then it becomes clear that the curves seen in the triangle, when considered in an enlarged frame, are part of one and only one continuous loop. This loop goes to, and returns from, infinity three times. Hence the critical line

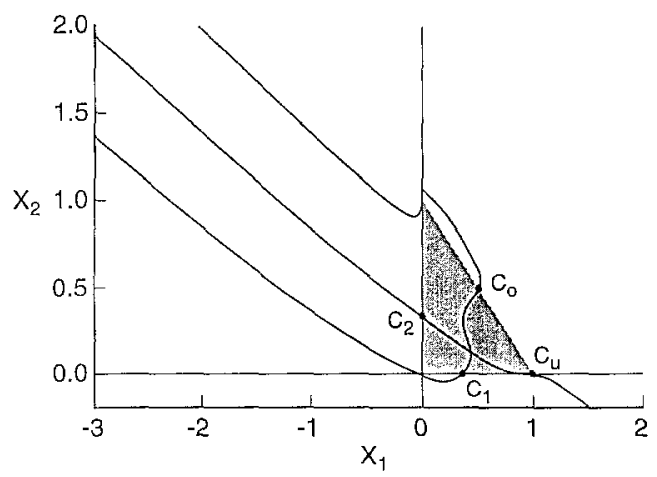

Fig. 1. Critical line of the van der Waals equation at the van Laar point extended outside the physical triangle. The critical points $C_{1}$ and $C_{2}$ represent the cases of pure component 1 and pure component 2 , respectively, $C_{0}$ the critical mixing in the absence of holes, and $C_{u}$ the end of the unstable part. 


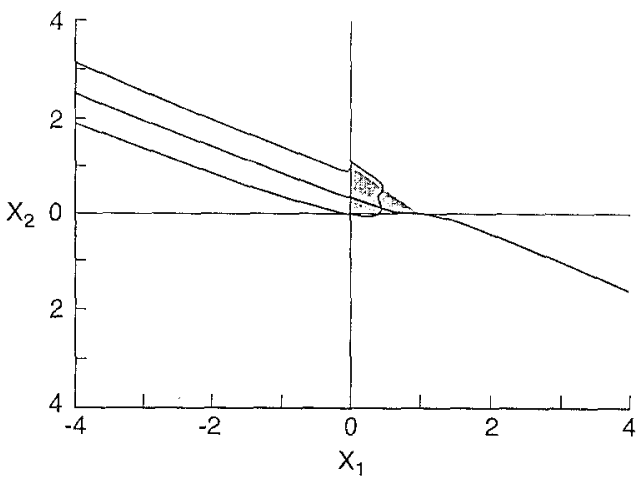

Fig. 2. Zoom away from Fig. 1, showing the asymptotes.

intersects itself three times at infinity; this is a mathematical triple point. Admittedly, there is no physical use for an isolated point at infinity; this is merely a way of expressing the fact that the number of asymptotes turns out to be less than the number of roots of the highest power of the polynomial, which made further zooming unnecessary. However, it can never be excluded that such a point is "activated" when the equation is generalized.

Plotting the curve outside the triangle of physical interest (Fig. 1) shows three asymptote branches in the upper left sector, but only one in the lower right section. Looking at larger distances (Figs. 2 and 3) reveals the missing pieces in the lower left corner of our "zoomed" picture (Fig. 3).

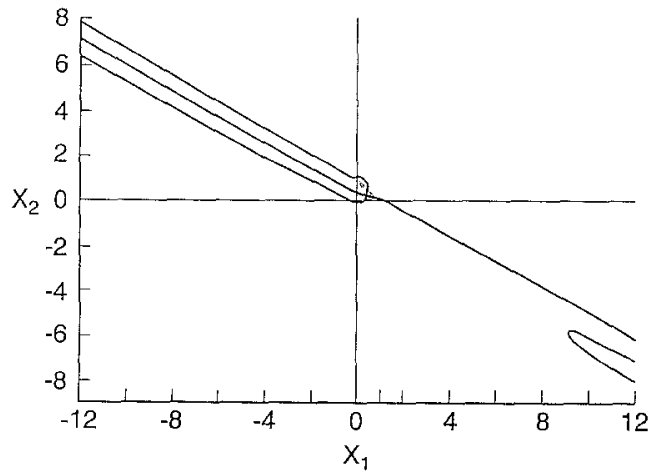

Fig. 3. Further zoom, showing the missing branches of the asymptotic structure in the lower left corner. 


\section{THE VAN LAAR CRITICAL LINE IS IRREDUCIBLE}

The question arises whether the polynomial $F_{2}$ defining the van Laar critical curve can be factored again if one admits additional algebraic numbers in the coefficients. We shall prove that no such factorization is possible over the complex numbers. (In algebraic geometry such a curve is called "absolutely irreducible.") The proof is indirect. Suppose that

$$
F_{2}(u, v)=g(u, v) h(u, v)
$$

where $g(u, v)$ and $h(u, v)$ are nonconstant polynomials in $u, v$ with real or complex coefficients. Now we substitute $v=(1+\sqrt{3}) u+c$ in (7). Here $c$ is a new variable. One computes

$$
\begin{aligned}
& F_{2}(u,(1+\sqrt{3}) u+c) \\
&= {\left[18 c^{3}-(27+27 \sqrt{3}) c^{2}-(9+9 \sqrt{3}) c+(63+36 \sqrt{3})\right] u^{2} } \\
&+\left[(-60+12 \sqrt{3}) c^{3}+36 \sqrt{3} c^{2}+(72+48 \sqrt{3}) c\right] u \\
&+\left[(60-20 \sqrt{3}) c^{3}+36 c^{2}\right]
\end{aligned}
$$

Let us denote by $\widetilde{F}(u, c)$ this polynomial in $u, c$. Now, $\widetilde{F}(u, c)$ is a quadratic polynomial in $u$. Similarly, we define

$$
\tilde{g}(u, c)=g(u,(1+\sqrt{3}) u+c), \quad \tilde{h}(u, c)=h(u,(1+\sqrt{3}) u+c)
$$

Can $\tilde{g}(u, c)$ be independent of $u$ ? If so, $\tilde{g}(u, c)=\tilde{g}(0, c)$. First suppose that the last member is also independent of $c$. Then $\tilde{g}(u, c)$ is independent of $u$ and $c$. Then substituting $c=v-(1+\sqrt{3}) u$ in $\tilde{g}(0, c)$, one sees that $g(u, v)$ does not depend on $u, v$. This is a contradiction. Hence $\tilde{g}(0, c)$ is a nonconstant polynomial in $c$. Then there exists some real or complex number $\gamma$ such that $g(0, \gamma)=0$, which implies that $\tilde{F}(u, \gamma)$ is the zero polynomial. Hence $F_{2}(u,(1+\sqrt{3}) u+\gamma)$ is the zero polynomial. This means that the coefficients of $u^{2}, u^{1}, u^{0}$ in (8) vanish simultaneously for $c=\gamma$. It is easy to see that no such $\gamma$ exists. So we have proved that $\tilde{g}(u, c)$ depends on $u$. The same argument applies to $\tilde{h}(u, c)$. So we have obtained a factorization of the polynomial $\widetilde{F}(u, c)$, quadratic in $u$, as a product of the two polynomials $\tilde{g}(u, c), \tilde{h}(u, c)$, both containing $u$. As is well known, such a factorization is possible if and only if the discriminant of $\widetilde{F}$ as a polynomial in $u$ is a full square (of a rational expression in $c$ ). This discriminant equals $a / b$, where

$$
\begin{aligned}
a= & -144 c^{2}\left[4 c^{7}-(6+6 \sqrt{3}) c^{6}-(38+20 \sqrt{3}) c^{5}\right. \\
& +(77+49 \sqrt{3}) c^{4}+(291+171 \sqrt{3}) c^{3} \\
& \left.+(33+20 \sqrt{3}) c^{2}-(435+251 \sqrt{3}) c-(291+168 \sqrt{3})\right] \\
b= & 2 c^{3}-(3+3 \sqrt{3}) c^{2}-\sqrt{3} c+(7+4 \sqrt{3})
\end{aligned}
$$


Since $a$ and $b$ are polynomials of odd degree having no common factors containing $c$, it is obvious that $a / b$ is not the square of a rational function in $c$. This completes the proof that $F_{2}$ is an absolutely irreducible curve.

The following remark may be of interest to mathematically minded readers: the van Laar critical curve has genus $1 .^{(11)}$ We do not need or use this fact and we shall not give a proof (it is an easy consequence of Plücker's formula). Loosely speaking, a plane algebraic curve of genus 1 is a curve that cannot be rationally parametrized (as, for instance, conic sections), but admit a parametrization by elliptic functions. Elliptic functions are met in many domains of physics, e.g., the exact motion of the simple pendulum.

\section{THE SECOND BRANCH}

In calculating the critical lines at the van Laar condition, the second root of the Hessian has been ignored so far. This root corresponds to a solution with $T=0$. This is not typical for the van Laar parameters; the result occurs for any set of parameters that fulfills the geometric mean condition: $U_{0}=-U_{1} U_{2} /\left(U_{1}+U_{2}\right)$. This extra branch has no physical use, since not only $T=0$, but also the corresponding lines are completely unstable. It is nevertheless worthwhile to inspect these lines since for nongeometric mean conditions they will be coupled to the first branch. That is to say, the point where the first and second networks do cross may or may not open up when a slight deviation of the geometric mean condition is made.

In Fig. 4 we show the superposition of the two networks, and in Fig. 5 the second net only. The general van der Waals equation always has a

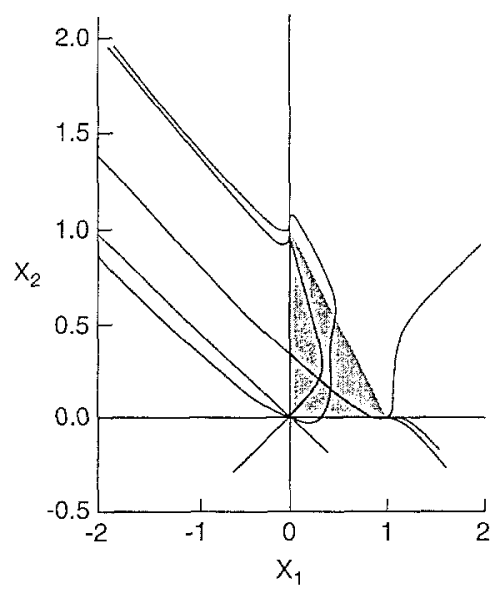

Fig. 4. First and second networks superimposed. 


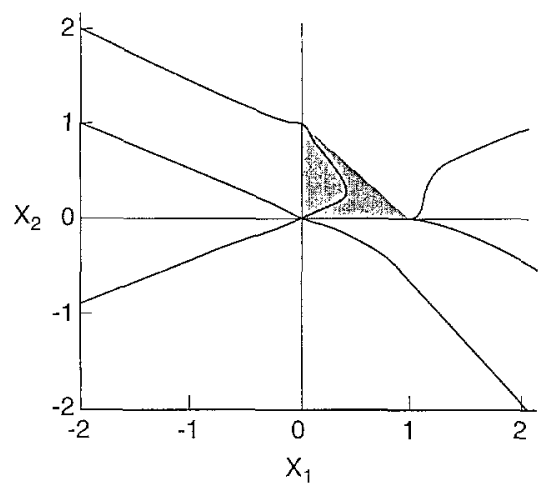

Fig. 5. Second branch only, on an expanded scale.

solution at all three corners. As can be seen from the figures, the behavior near each of the three corners is entirely different. The first branch goes through the lower left-hand corner, without ever entering the triangle. The second branch enters at this corner, resulting in a critical line, unstable everywhere inside the triangle, which goes from the origin 0,0 to the upper corner 0,1 . Where the second branch leaves, through the top, it meets the first branch, which goes through this point also, with a vertical tangent, without ever entering. Finally, in the $(1,0)$ corner there is a passingthrough of the first network and a cusp point of the second network belonging to the branch that lies entirely outside the physical triangle. The last phenomenon is rather interesting, since it was found by Kraska and Deiters $^{(12)}$ that for the Redlich Kwong equation (a cubic equation of state of the van der Waals type), for certain values of the interaction parameters, a small loop starts to appear in this corner, which may be an outgrowth of this cusp (see Table $2, \lambda=-0.105$, in the above reference).

Figures 5 and 6 describe the asymptotes of the second net. It is only on the scale of Fig. 6 that one sees the two extra branches in the upper left and lower right quadrants appear, making up the complete set. Again this proves that no further factorization is possible; we deal with one continuous curve when the points at infinity are incorporated.

The reasons for observing the critical line beyond the normal stable segments are twofold. Consider again the unstable part inside the triangle (see Fig. 5); this "bulge" may be unstable here, but for totally different values of the interaction parameters, segments of this line will be connected to stable parts, so one can say that this line will eventually play a role. Finally, the use of parts outside may be unphysical, but it sometimes helps to make a point, as illustrated by the work of Levelt Sengers, ${ }^{(13)}$ who introduced negative concentrations to clarify the behavior near $x=0$. 


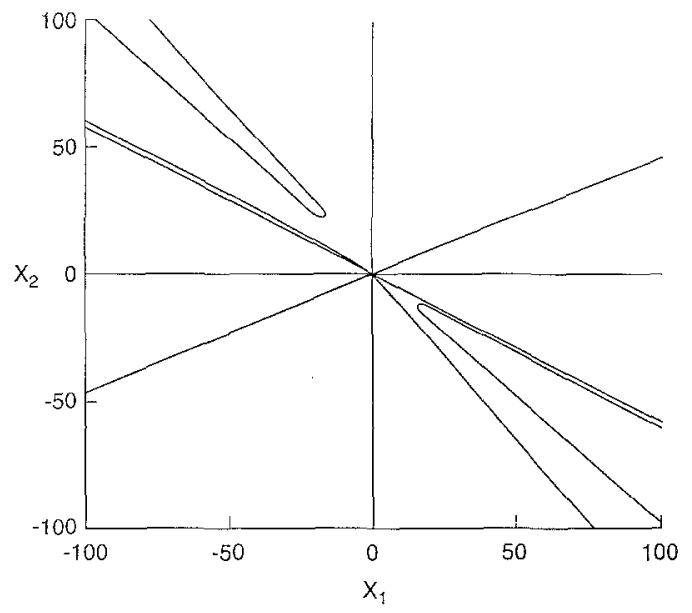

Fig. 6. Complete set of asymptotes of the second branch.

\section{BEHAVIOR NEAR $C_{0}$}

It is little known, but it may be also of little importance, that the behavior of the van der Waals equation in the neighborhood of the critical point of the pure liquid-liquid separation is rather different from that of the lattice gas equation in this region. That is, the asymptote in the $p-T$ projection approaches the vertical line from the opposite side. This can be seen as follows.

The Gibbs free energy surface, at fixed temperature, has areas of positive curvature and areas of negative curvature. The dividing lines, the loci of zero curvature, are, in terms of physics, the spinodals. Since the critical point is part of the spinodal, we can use the line segment of the spinodal in the immediate neighborhood of this point as a "poor man's" approximation to the binodal. This is done since the spinodal can be determined easily by algebraic means, while the binodal cannot.

Rather than use the van Laar parameters, we will choose a set of parameters used by Scott and van Konynenburg (ref. 1, Fig. 7; $\zeta=0.473$, $A=0.105$ ) in order to magnify the effect, since the result is not restricted to the van Laar case. For a temperature between $T^{\infty}$ and $T_{\text {UCEP }}$ (the upper critical end point temperature), the tie-line diagram has both a triple point and a critical point. As an example we reproduced one figure from the set of tie-line diagrams used by Knobler and $\operatorname{Scot}^{(14)}$ to describe ternary mixtures of $\mathrm{CO}_{2}$ with methanol and ethane at a given temperature and pressure. (Note: in a ternary diagram of this type $p$ and $T$ are fixed, but 


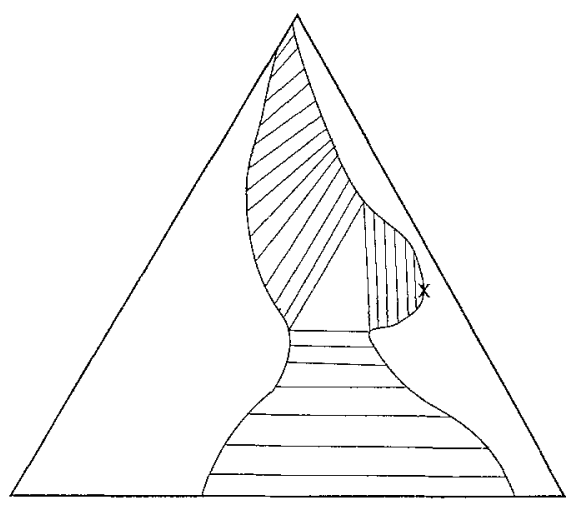

Fig. 7. Tie-line diagram of a ternary system which has both a triple point and a critical point at a given temperature and pressure.

when applied to a binary gas-liquid system only $T$ is fixed, since the pressure will determine the number of holes, the third component). Another example is found in Scheffer, ${ }^{(15)}$ where both the binodals and the spinodals are drawn in Fig. 179 on p. 271, a $V-x$ diagram. In Fig. 7 one notices a triangle in the center; this represents the three-phase point for that temperature. Two sides of the triangle are flanked by a set of tie-lines which eventually reach the boundaries: $x_{1}=0$ and $x_{2}=0$; and the third side of the triangle has a set of tie-lines which end in a critical point (indicated with a cross), which lies close to the line $x_{1}+x_{2}=1$, the line of jamming points. It is taken for granted that this critical point will move, upon lowering of the temperature, toward this axis and meet it at the temperature $T^{\infty}$ given by $T^{\infty} / T_{1}=\left(U_{1}+U_{2}\right) / 4$ at the value $x_{1}=x_{2}=1 / 2$; however, this scenario is not quite correct in the case of the van der Waals equation for binary mixtures.

What actually happens can be seen more easily if the spinodal branch outside the physical triangle is taken into account. This branch, which is not present in the lattice gas model, also has a critical point. Using a parameter $d$ as the distance from the jamming line, $x_{1}=x_{2}=1 / 2+d$, we show the critical temperature $\left(\Delta T=T-T^{\infty}\right)$ dependence on this parameter in the insert of Fig. 8. Upon lowering of the temperature, the "inside" critical temperature will approach the axis, as expected, but the "outside" critical temperature will get there first. Upon further lowering of the critical temperature the critical points will meet and then disappear by the usual branch exchange mechanism of the spinodals. When this is plotted in a $p-T$ diagram one finds that the asymptote approaches from the 


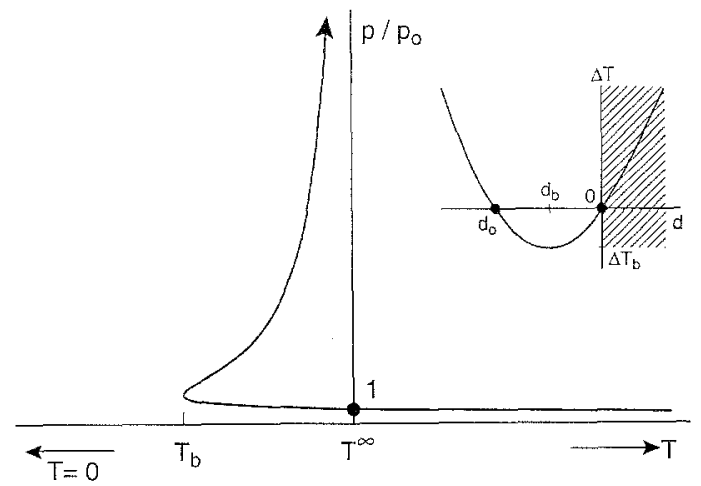

Fig. 8. Liquid-liquid demixing at high pressures. The asymptote approaches from the left after crossing the vertical. Insert: the critical temperature as a function of the distance from the point $x_{1}=x_{2}=1 / 2$.

left after passing through the temperature $T_{b}$ ( $b$ for bulge). Using the van der Waals equation, we find that the pressure is approximately proportional to $-d^{-1}$ in this region. The temperature range is very small. Numerical calculation for the Scott and van Konynenburg parameters that were mentioned above gives $\left(T_{b}-T^{\infty}\right) / T^{\infty}=-0.012$. The $p-T$ projection of the critical line in this region is plotted in Fig. 8. The origin $T=0$ lies far to the left and the three-phase line, not shown, lies below the curve. To the right the critical line will eventually join the three-phase line at $T_{\text {UCEP }}$.

If we change the value of $\sigma$ to zero in Eq. (1), we obtain the lattice gas model and the asymptote approaches from the right. The unphysical branch disappears in the jamming line. Numerical work shows that the changeover from one model to the other occurs already at very small values of $\sigma$ compared to one.

\section{CONCLUSION}

Further reduction of the polynomial representing the critical line in the van Laar case would have been useful in order to obtain a mathematically compact result. The result of this investigation is that this is not possible. Since the van der Waals equation is the simplest aong the cubic equations of state, it also shows that it is unlikely to happen for other equations as well. On the other hand, the method of going outside the physical region may have some merits in the study of modifications of the equation of state. 


\section{ACKNOWLEDGMENTS}

We like to thank Dr. I. L. Pegg for many discussions and for plotting the curves. One of us (P.H.E.M.) thanks Drs. Richard F. Kayser and Michael R. Moldover, both of the Thermophysics Division at NIST, for their hospitality.

\section{REFERENCES}

1. R. L. Scott and P. H. van Konynenburg, Disc. Faraday Soc. 49:87 (1970); P. H. van Konynenburg and R. L. Scott, Phil. Trans. R. Soc. 298:495 (1980).

2. D. Furman and R. B. Griffiths, Phys. Rev. A 17:1139 (1978).

3. D. J. Korteweg, Arch. Néere. Sci. Ex. Nat. II VII:235 (1903); K. Akad. Amst. 5:445-465 (1903).

4. A. van Pelt and Th. W. de Loos, J. Chem. Phys. 97:1271-1281 (1992).

5. A. van Pelt, C. J. Peters, and J. de Swaan Arons, J. Chem. Phys. 95:7569-7575 (1991); A. van Pelt, Critical phenomena in binary fluid mixtures; Classification of phase equilibria with the simplified-perturbed-hard-chain theory, Thesis, Technical University of Delft (1992).

6. J. J. van Laar, Proc. Sect. Sci. K. Ned. Akad. Wet. 7:646 (1905); 8:33 (1905).

7. E. P. van Emmerik, J. J. van Laar (1860-1938), a mathematical chemist, Thesis, Technical University of Delft (1991).

8. U. K. Deiters and I. L. Pegg, J. Chem. Phys. 90:6632-6641 (1989).

9. P. H. E. Meijer, I. L. Pegg, J. Aronson, and M. Keskin, The critical lines of the van der Waals equation for binary mixtures around the van Laar point, Fluid Phase Equilibria 58:65-80 (1990).

10. P. H. E. Meijer, The van der Waals equation of state around the van Laar point, J. Chem. Phys. 90:448 (1989).

11. Robert J. Walker, Introduction to Algebraic Curves (Princeton University Press, Princeton, New Jersey); W. Fulton and R. Weiss, Algebraic Curves, An Introduction to Algebraic Geometry (Benjamin/Cummins, Menlo Park, California, 1969).

12. Th. Kraska and U. K. Deiters, J. Chem. Phys. $96: 539$ (1992).

13. J. M. H. Levelt Sengers, Thermodynamics of solutions near the solvent's critical point, in Supercritical Fluid Technology, Th. J. Bruno and J. F. Ely, eds. (CRC Press, Boca Raton, Florida, 1991), Chapter 1.

14. C. M. Knobler and R. L. Scott, in Phase Transitions and Critical Phenomena, Vol. 9 , C. Domb and J. L. Leibowitz, eds. (Academic Press, 1984), p. 163.

15. F. E. C. Scheffer, Heterogene Evenwichten in Unaire and Binaire Stelsels, 2nd ed. (Uitg. Waltman, Delft, 1960). 